\title{
Subgrade strengthening of roads and railroads as planar geotextile reinforcement
}

\author{
Krzysztof Gradkowski ${ }^{1, *}$ \\ ${ }^{1}$ Warsaw University of Technology, Civil Engineering Department, Al. Armii Ludowej 16, Poland
}

\begin{abstract}
The proper quality of construction of roads and railroads can be achieved by strengthening the of the foundation bed. Measurements and tests the number and order of the layers of geotextile layer of ground indicate the effectiveness of technical solutions involving the reinforcement laid horizontally. The offer technical use of the geotextile reinforcement in the ground layer substrates of roads indicates the possible effectiveness of the strengthening of the subsoil of roads and is also included in this article.
\end{abstract}

\section{Introduction}

In practice, the implementation of the sections of the transport infrastructure is the need to improve and strengthen the ground layer of pavement surfaces of roads and railroads. In the case of semi-rigid surface highways are layers adjacent to the substructure. At the European railway routes it is envisaged building the so-called. ground the protective layer. In both cases, the use of geotextile layers into ground layers is an alternative to the widespread use of hydraulic binders (cement, lime). By installing geotextile layers in the subsoil of roads and railroads also we obtain as well;

- proper strengthening layer substrate surface,

- favourable changes in soil moisture in the surface layers of the substrate,

- reducing the cost of construction in the area of substrates surface.

These effects are due to the reinforcing properties of geotextiles interaction with the soil substrate. The scope of strengthening the structural layer of the ground floor surface of roads depends on several factors. Some of them have been identified on the basis of laboratory testing measurements.

\section{Tests measuring reinforced ground with geotextiles}

Since the beginning of the twentieth century saw rapid growth applications of geosynthetic materials in infrastructure construction. The use of geosynthetic materials and geotextile in round constructions increases the stability and durability and stability, especially road infrastructure construction [1]. Different types of geotextile expose the different properties, whose effective use in the construction of roads is the subject of specific research programs

${ }^{*}$ Corresponding author: k.gradkowski@il.pw.edu.pl 
$[2,3]$. Commonly known and used are properties of filtration and separation geotextiles. Effective and tangible research results obtained in the measurement chamber, beyond the actual sections of roads as well. Laboratory research programs on the use of geotextiles are economical and indicate the direct technical solutions [4].

\subsection{Preliminary oedometer test}

As part of the preliminary measurements of signal preparation for wider research, made several signalling series, standard measurements oedometer test, according to two schemes fulfilling the oedometer barrel, fig.1. A series of measurements performed for medium granular sand (MSA) of natural humidity of about $10 \%$. The study, performed by Polish standards PN-88 / B-04481, is used laboratory oedometer test with a range of loads $\boldsymbol{p}$ and $\boldsymbol{p}_{z}$ from 12.5 to $400 \mathrm{kPa}$ and a sample height of $20 \mathrm{~cm}$.
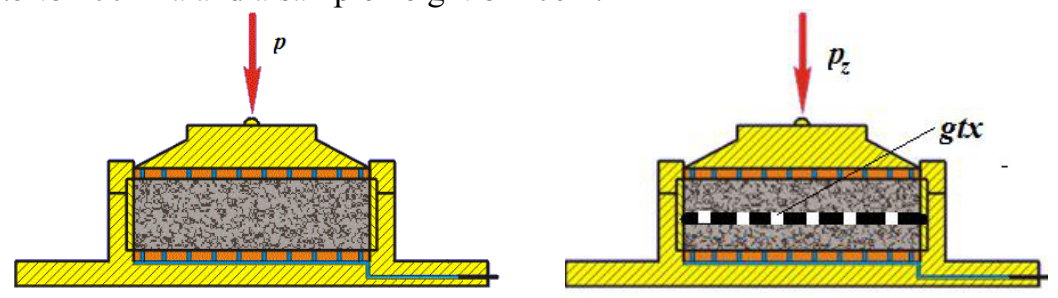

Fig.1. Schemes of fulfil and oedometer loading.

Repeated sequences of oedometer measurements and statistical results approximations, allowed us to obtain the graph of figure 2 .

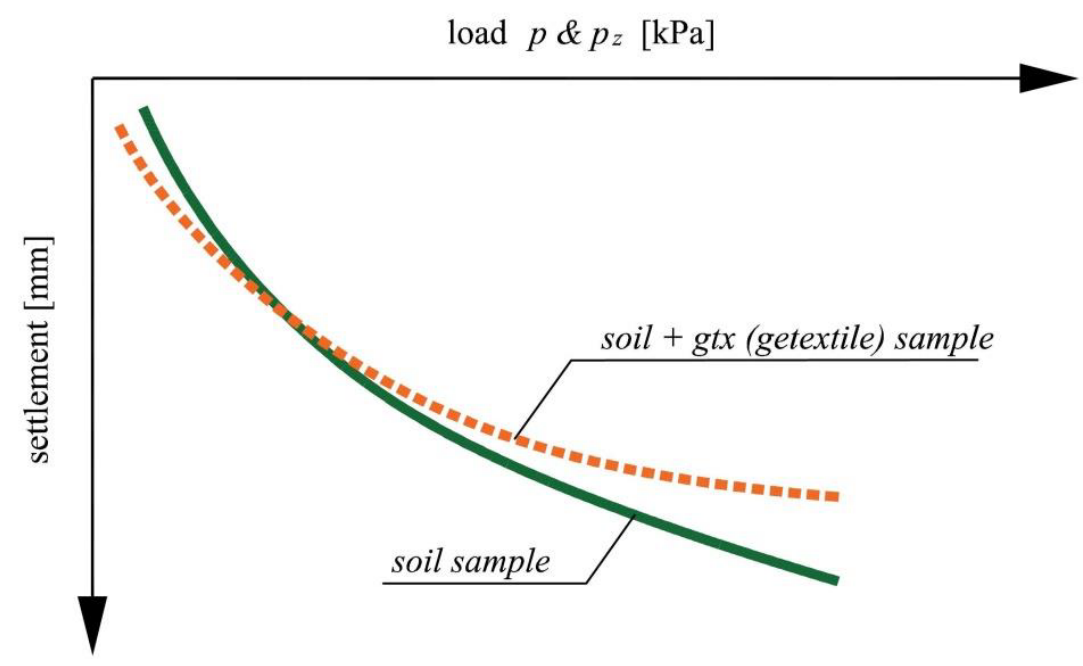

Fig.2. Statistical performance the measurements of two ways of filling oedometer barrel.

The graph in figure 2 clearly shows a tendency to strengthen the soil through the geotextiles reinforcement with one layer. The course of the curves is an approximations not less enhancement effect by the geotextile is very clear. 


\subsection{Oedometer test program}

In order to perform direct testing laboratory prepared samples appropriate forms to fill different structures stratification geotextiles, one type of sandy soil with known physical parameters. Land filling was a medium sand, $\rho=1.78 \mathrm{~g} / \mathrm{cm}^{3}$, solid interior humidity $=5 \%$ and the particle size uniformity index $\mathrm{C}_{\mathrm{U}}=5$. Methods roller made of non-deformable sections of the tube height $\mathrm{H}=25 \mathrm{~cm}$ and an internal diameter $\mathrm{d}=10 \mathrm{~cm}$, that is, with a coefficient of proportionality, the shape of the sample of 2, 5. Used in the research commonly used in road geotextile having a basis weight of $200 \mathrm{~g} / \mathrm{m}^{2}$. In the six forms of the samples were performed four series fills the ground with different structures stratification geotextile in each sample, Figure 3. In the next series A, B, C and D were both variable number of layers of geotextile $\mathrm{N}$, and the distance between the layers of geotextile. In each series was made a comparative sample only filled with soil. The series $\mathrm{C}$ and $\mathrm{D}$ geotextile used in a double arrangement, thereby obtaining a basis weight of $400 \mathrm{~g} / \mathrm{m}^{2}$. Soil-geotextile sample preparation system shown in figure 3

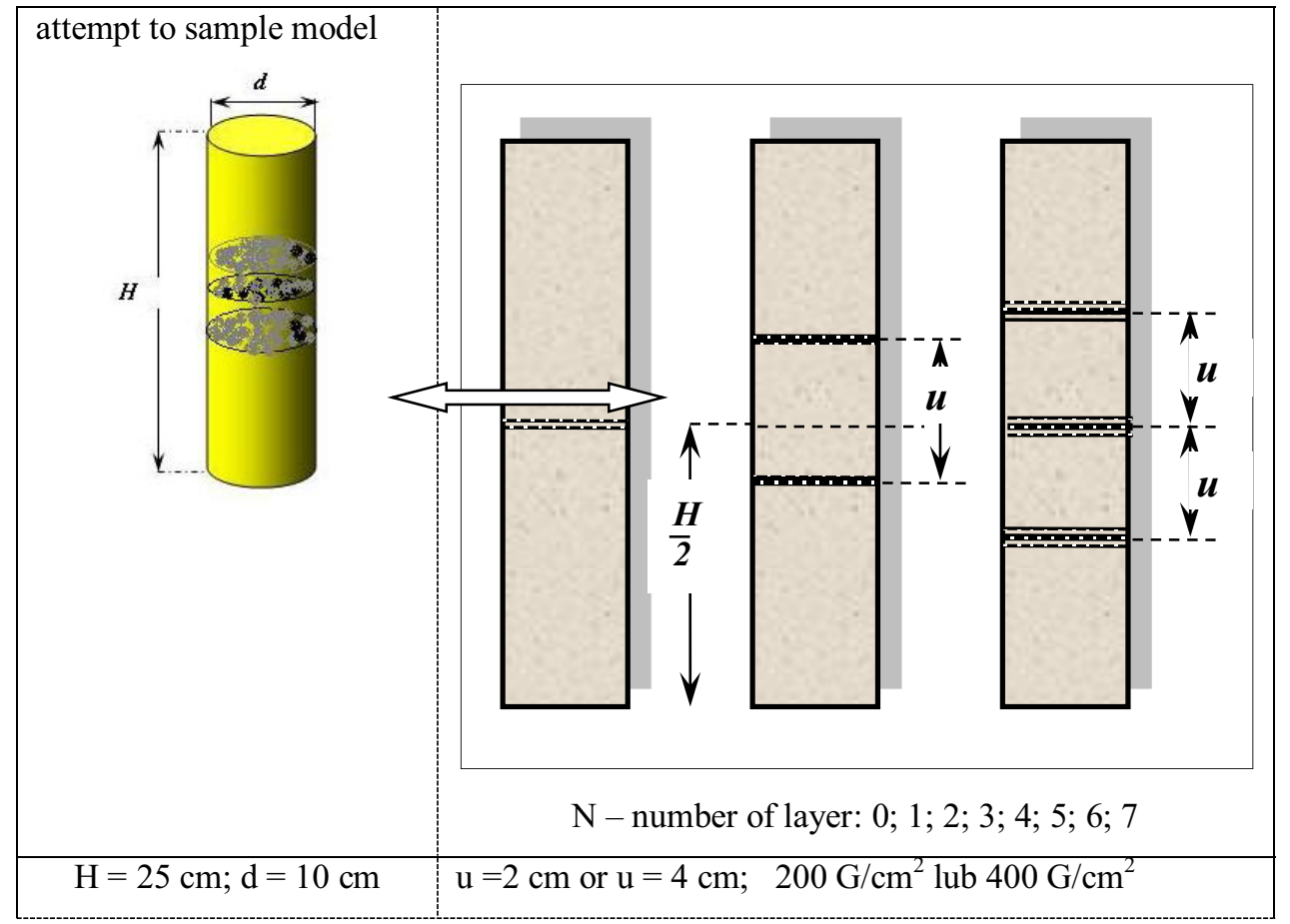

Fig. 3. The structures of preparing a series of samples having a variable number of $\mathrm{N}$ layers at distances of $u$.

Determine the extent and scale of load and displacement were made on a single sample zero, filled with the soil compacted layers in the manner adopted for all samples. Before you can record movements, in order to harmonize the conditions for direct measurement and maximum elimination of differences in the density of filling the ground, each of the samples were subjected to pre-load of $100 \mathrm{kPa}$. The load was single, and after unloading the sample was applied as the initial load. The expected layout changes susceptibility of the tested samples, depending on the increase in the number of layers of geotextiles. Susceptibility to deformation of the sample that is, changing the height of the sample is defined as the reciprocal of the spring constant of the sample and may be a measure of the compressive strength of the laminar structure of the ground geotextile - layer. Testing 
machine is equipped with a digital recorder and monitor graphical image measurement of strains and loading forces fig. $4,5$.

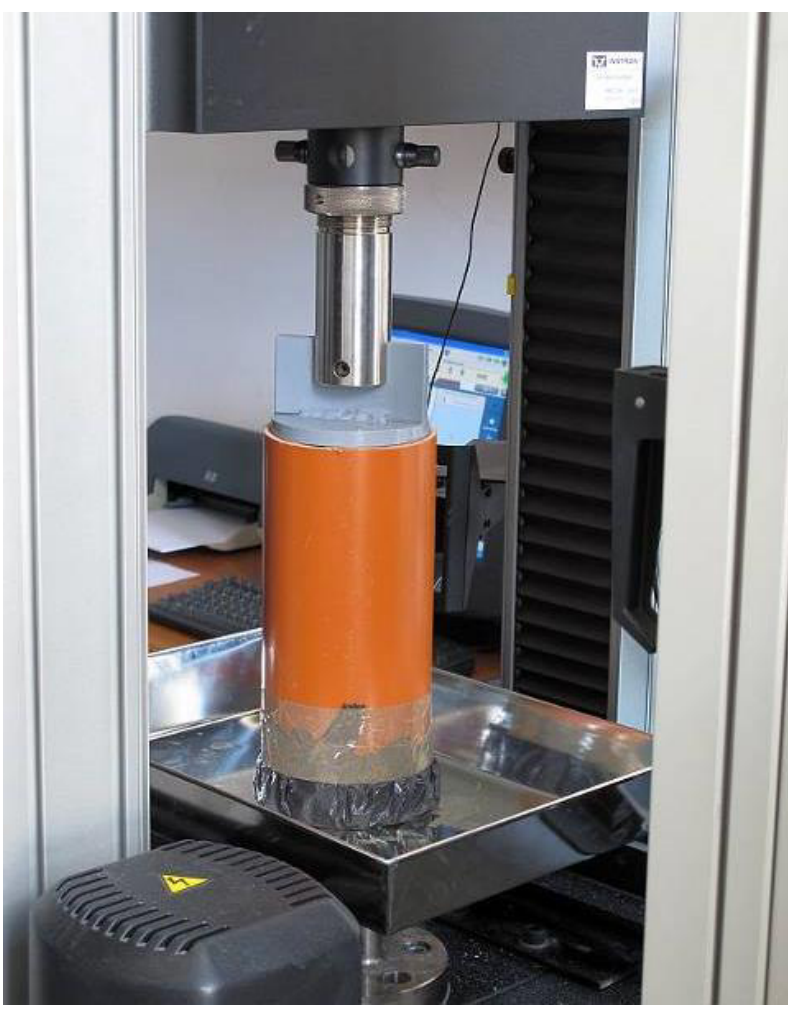

Fig. 4. Uniaxial load filled with samples.

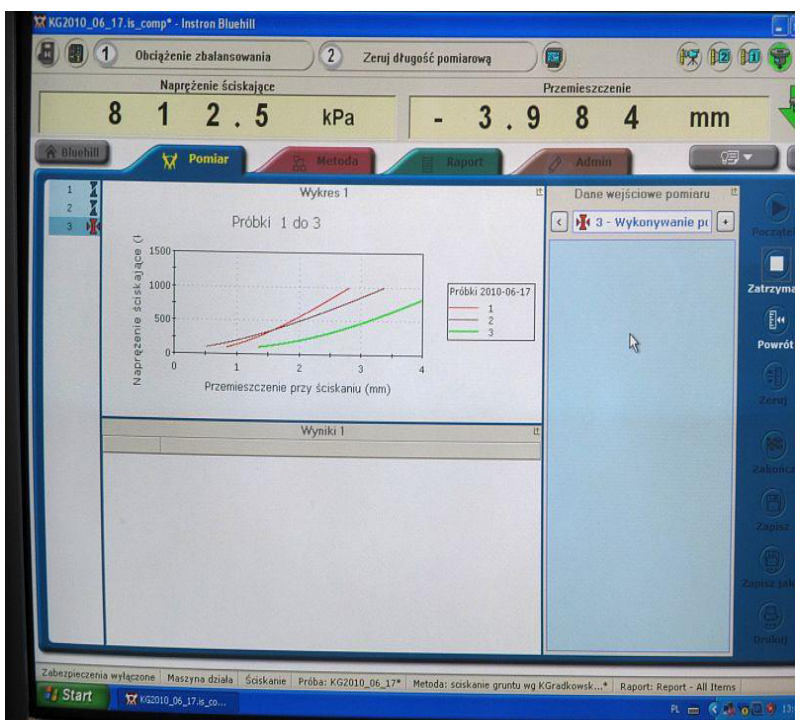

Fig. 5. Screen registrar.

Summary of results and presented in graphs the results of direct measurement of susceptibility samples of each series. And a graphical summary of these results fig. 6 is of 
some importance to determine the appropriate way to strengthen planar geotextile ground reinforced. The analysis results were not taken into account the extreme that could arise as a result does not maintain uniform conditions for the preparation of all samples or difficult to avoid errors in the care of the samples.

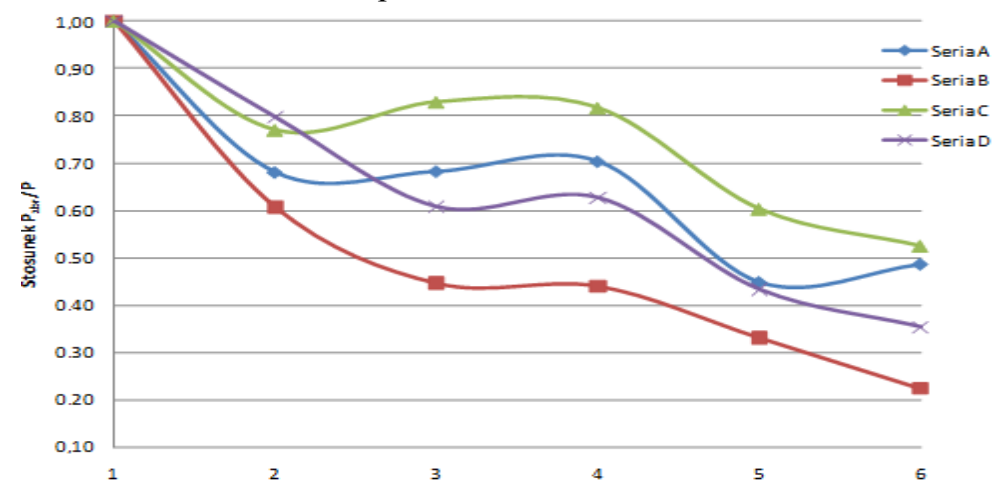

Fig. 6. Graphical comparison of the settlement susceptibility of the samples series A, B, C, D.

\section{Conclusions and scope of applications}

Generalizations of the specific proposals from the conducted test sequence and measurements referred to from [4], lead to the following statements:

$>$ Subgrade and subbase ground layers can be reinforced with geosynthetics including geotextiles and geogrids. The use of these give a significant strengthening effect of the substrate road surface, a larger modulus of the multilayer substrate surface and reduces the susceptibility to permanent deformation and settlement.

$>$ The use of several layers of geotextiles resulting in a material gain range of multilayer substrate surface highways and rail roads, and does not limit their functions separation and filtration in a multi-layer system, ground substrates foundation of roads and railroad.

$>$ Single-layer geotextiles reinforcement is not a sufficient reinforcement of the substrate surfaces of highways, only retain their function layer separation and filtration function circuit substrate. Number and order of geotextile layers in the soil-ground mobilizes interaction and friction of the ground layer strengthening.

$>$ An effective form of reinforcement layers of soil substrate surface is to use two or three layers of geotextiles, the total thickness of the reinforced layer of primer should not exceed $25 \mathrm{~cm}$ significantly greater enhancing effect layers can be obtained by replacing the geogrid of one of the layers of geotextile.

$>$ The appropriate thickness of the soil layer between the sandy layers of geotextiles depends on the parameters of the quality of the land and should not exceed $10 \mathrm{~cm}$ while maintaining the required densities.

$>$ Strengthening and reinforcement geosynthetics ground floor paving of roads is an alternative design for the reinforcement and improvement of ground surface using hydraulic binders and can be economically competitive. 


\section{References}

1. J.G. Zamberg, R. Gupta, 9th International Conference on Geosynthetics, Brazil, 380398 (2010)

2. M.S.N. Tafreshi, A.R. Dawson, Geotextiles and Geomembranes 28, 72-84 (2010)

3. E. M Palmira, Geotextiles and Geomembranes 27, 368-390 (2009)

4. K. Gradkowski, Testing ground subgrade reinforced with geosynthetics. (Publishing House WUT, Warsaw, 2013) 